\title{
PARA UNA TEORÍA DE LA ARGUMENTACIÓN JURÍDICA
} ay por lo menos tres perspectivas (relativamente distintas entre sí) desde las que puede examinarse una teoría de la argumentación jurídica. La primera se refiere a su contenido o campo de aplicación. Quien se sitúa en esta perspectiva y se plantea, en consecuencia, la cuestión de qué es lo que explica la «teoría estándar de la argumentación jurídica» (y con este concepto pretendo aludir a la teoría elaborada por autores como Aulis Aarnio, Robert Alexy, Neil MacCormick o Aleksander Peczenik, para citar a los que cabe considerar como más influyentes en la última década) podría contestar diciendo que todas ellas (con diversos grados e intensidades) son teorías que estudian los aspectos normativos (entendiendo por tal la argumentación que se contiene en los «fundamentos de Derecho») de la justificación o fundamentación de las decisiones tomadas por órganos judiciales situados en los niveles más altos de la administración de justicia. De aquí resultan también una serie de críticas que se podrían dirigir a la teoría estándar de la argumentación jurídica: Por ejemplo, podría dudarse de que en este campo tenga sentido (es decir, resulte fructífera) la distinción entre contexto de descubrimiento y contexto de justificación, y así se podría reprochar a estos autores el haber construido teorías normativas (no en el sentido de que se refieran a normas, sino en el de que proponen o prescriben normas) de la justificación de las decisiones que, sin embargo, no pueden dar cuenta del proceso real de la fundamentación; es decir, no es sólo que no se explique cómo se llega a una decisión, sino que tampoco se da cuenta de cómo se fundamenta de hecho la decisión tomada. Podría criticárseles también el no haber prestado atención a los aspectos empíricos de la argumentación, es decir, a la argumentación ligada no con normas jurídicas, sino con hechos (por ejemplo, la argumentación en materia de prueba). El haber descuidado el estudio de la
\end{abstract}


argumentación que tiene lugar en los tribunales inferiores o incluso en órganos que no poseen carácter judicial. $\mathrm{O}$, en fin, el no haber considerado el hecho de que no se argumenta sólo en el proceso de interpretación y aplicación del Derecho, sino también en el de su establecimiento.

La segunda perspectiva se refiere a los métodos o instrumentos utilizados por la teoría estándar; por lo tanto, la cuestión no es ya: ¿qué es lo que explica la teoría?, sino ¿cómo lo explica? Un crítico situado en esta perspectiva podría hacerse la siguiente reflexión: Concedamos que lo que debe explicar una teoría de la argumentación jurídica sea precisamente lo que explica la teoría estándar. ¿Pero lo explica bien? ¿No cabría plantear las cosas de otra manera, es decir, utilizar otros instrumentos conceptuales, diferentes a los manejados por estos autores? ¿Cuáles serían esos métodos?

Finalmente, la tercera perspectiva concierne a los resultados obtenidos por la teoría estándar de la argumentación jurídica. Quien se sitúa en este punto de vista se plantea la cuestión de para qué sirve esta teoría. Las críticas que pueden dirigirse desde aquí pueden ser de dos tipos. Por un lado, puede ponerse en cuestión el valor instrumental de la teoría, por ejemplo, procurando mostrar que no tiene interés -o que tiene un interés muy limitadotanto para el práctico como para el dogmático del Derecho. Por otro lado, puede discutirse acerca de su valor ideológico; por ejemplo, podría aducirse que la teoría estándar lleva en realidad a justificar las formas de fundamentación dominantes en la práctica y en la dogmática jurídica (y ésta es, una objeción que quizás no sea incompatible con la anterior) $\mathrm{y}$, en el fondo, a la justificación del propio sistema jurídico de referencia, e incluso del Derecho en cuanto tal (o, si se quiere, de un tipo de Derecho: el Derecho de los Estados democráticos).

Antes he dicho que estas críticas no podrían aplicarse a todos los autores por igual. Ahora tengo que precisar que algunas de ellas no se les podría aplicar (por lo menos a algunos de ellos) en absoluto, lo que sin embargo no afecta a la validez del concepto de «teoría estándar de la argumentación jurídica». Esto es así porque el concepto en cuestión lo entiendo como un «tipo ideal» en el sentido de Max Weber; por lo tanto, no pretende tener un carácter descriptivo, sino un valor instrumental. Por ejemplo, a partir de él podría procederse a analizar las teorías de estos (o de otros) autores para comprobar si, y hasta qué punto, pueden dirigírseles las críticas sugeridas antes (que, por otro lado, no hay por qué dar por descontado que sean acertadas).

Aquí, sin embargo, no voy a pretender efectuar un análisis de 
semejante amplitud. Me contentaré con situarme en la segunda de las perspectivas señaladas, y con ocuparme -o empezar a ocuparme- de una de las críticas que podría dirigirse contra la teoría estándar y que me parece tiene una considerable relevancia. La crítica en cuestión es ésta: Para representar (a fin de analizar conceptualmente) la argumentación jurídica, la teoría estándar recurre a la lógica formal (bien se trate de la lógica proposicional o de la lógica de predicados, y bien se usen o no operadores deónticos), pero ésta, por sí sola, no constituye un instrumento adecuado para tal fin. Aunque aquí no voy a dedicarme a mostrarlo, doy por descontado que éste es un reproche (con independencia de que esté o no justificado) que sí se puede formular a todos y cada uno de los autores recordados al principio. $\mathrm{Y}$, en fin, creo que también puede aceptarse sin necesidad de mayores comentarios que esta cuestión está estrechamente conectada con otros problemas que se planteaban desde las otras perspectivas (por ejemplo, el no disponer de un instrumental adecuado para la representación de la argumentación puede tener que ver con el abandono de ciertos aspectos de la argumentación y con su utilidad o no utilidad para la práctica), lo que vendría a probar que se trata de una cuestión verdaderamente central para la argumentación jurídica.

\section{II}

Para empezar por el principio, podríamos retomar el ejemplo clásico de silogismo o inferencia lógica: «Todos los hombres son mortales; Sócrates es un hombre; luego, Sócrates es mortal». Con independencia de que lo anterior lo entendamos como una regla de inferencia (o, mejor, como la aplicación de una regla de inferencia), a la manera de la silogística tradicional, o bien como una proposición de la forma «si... y... entonces...» a la manera de Aristóteles [cfr. G. Patzig, 1959, págs. 13 y sig.], y de que lo representemos o no en la notación de la lógica proposicional $([\mathrm{p} \rightarrow \mathrm{q}) \wedge \mathrm{p}] \rightarrow \mathrm{q})$ o de predicados $(\wedge \mathrm{x}[\mathrm{Px} \rightarrow \mathrm{Qx}$ $\wedge \mathrm{Pa}] \rightarrow \mathrm{Qa}$ ), hay algo -o más que algo- en todo lo anterior que resulta insatisfactorio. Por supuesto, que todos estamos de acuerdo en que si es cierto que «todos los hombres son mortales» y que «Sócrates es un hombre», entonces también tiene que ser necesariamente cierto que «Sócrates es mortal». La noción lógica de argumentación sirve, por tanto, para indicar que hay (o que no hay) una conexión necesaria o de deducibilidad entre unas proposiciones (las premisas) y otra u otras (la conclusión). Pero pa- 
rece que esto no es suficiente para reconstruir lo que habitualmente entendemos por argumento o por buen argumento. Por ejemplo, según lo anterior cabría construir también argumentos (buenos argumentos, desde el punto de vista lógico) con premisas falsas [en realidad, por lo anteriormente dicho, la silogística tradicional no considera, o bien excluye, la posibilidad de que las premisas sean falsas, mientras que Aristóteles se refiere muchas veces a conclusiones válidas efectuadas a partir de premisas falsas; cfr. Patzig, ibid.], o bien con premisas que contienen información no suficientemente comprobada: «Todas las mujeres griegas respetan a sus maridos; Xantipa es una mujer griega; luego Xantipa respeta a su marido»también sería entonces un argumento válido, aunque no parece que sea tan cierto que Sócrates haya sido respetado por su mujer.

Hay sin embargo todavía otra (al menos otra) razón por la que el modelo puramente lógico de argumento no resulta satisfactorio [cfr. Quesada, 1986, págs. 113 y sigs.; una crítica clásica a la concepción deductiva de la argumentación que aquí tomo muy en cuenta es la de Toulmin, 1958]. Y la razón es que nadie se lanza sin más ni más (sino que solamente lo hace en contextos muy especiales) a construir silogismos. Dudaríamos incluso de la salud mental de alguien con quien nos tropezáramos por la calle y nos recibiera con un: «Todos los hombres son mortales; Sócrates es un hombre; luego Sócrates es mortal», o bien con un saludo del tipo siguiente: «Cada vez que me encuentro con usted siento una gran alegría; ahora me acabo de encontrar con usted; por lo tanto siento una gran alegría.» Podríamos decir que entendemos lo que se nos dice (entendemos el significado de las palabras y de las proposiciones), pero realmente no le encontramos sentido a lo que se nos dice (salvo, por ejemplo, que supiéramos que la persona en cuestión está estudiando lógica y que, por tanto, no pretende propiamente argumentar, sino mostrar sus conocimientos sobre lógica). Argumentar, para decirlo claramente, es un acto de lenguaje que sólo cabe efectuar (en sentido propio) en determinadas situaciones; concretamente, en el contexto de un diálogo (con otro o con uno mismo), cuando aparece una duda o se pone en cuestión un enunciado, y aceptamos que el problema ha de resolverse por medios lingüísticos (por tanto, sin recurrir a medios como la coacción física [Lorenzen, 1973]).

Para que el ejemplo tradicional de silogismo podamos interpretarlo realmente como un argumento, tenemos que ser capaces de concebir una situación en que alguien se cuestiona (o cuestiona a otro) si Sócrates es mortal habida cuenta de sus excepciona- 
les virtudes, muy superiores a las de los hombres «de carne y hueso». Entonces sí que cobran sentido las premisas y la conclusión pues, en efecto, si las premisas son verdaderas, entonces la solución para nuestro problema (la conclusión) no admite ya duda: Sócrates es mortal, y nuestro diálogo o nuestra reflexión interior puede continuar a partir de aquí. El argumento en cuestión podemos ahora representarlo así:

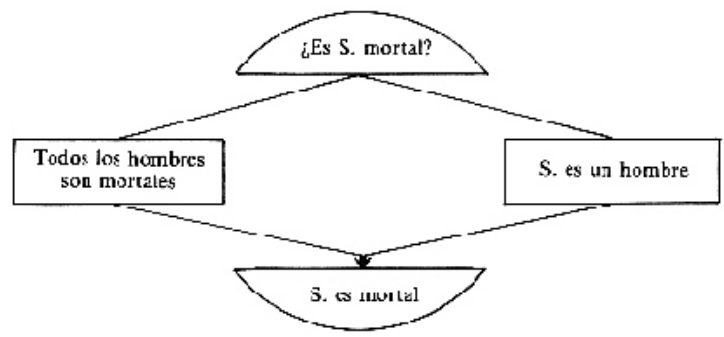

Y ello lo interpretamos de esta forma: la conjunción de las proposiciones «todos los hombres son mortales» $\mathrm{y}$ «Sócrates es un hombre» es un argumento en favor de la afirmación de que «Sócrates es mortal».

Sin embargo, no siempre (mejor dicho, casi nunca) las cosas son tan simples. Un argumento como el anterior presupone una situación (que quizás sólo se dé en los libros de lógica) en la cual la información que nos ofrecen las dos premisas no suscita duda alguna. Argumentar se reduce entonces a recordar (si se quiere, a ordenar) algunas cosas; pero, por supuesto, al final del proceso no puede decirse que hayamos obtenido nada nuevo. Ahora bien, los problemas para los que los hombres suelen necesitar ( $\mathrm{y}$ a veces utilizan) la argumentación son de un género bastante más complejo. En realidad, cabría decir que la situación anterior es una situación límite. Si concebimos el proceso de argumentar como el paso de unas informaciones (las premisas) a otras (la conclusión), la situación anterior se caracterizaría porque desde el comienzo (es decir, al plantear el problema) disponemos ya de toda la información necesaria y suficiente para llegar a la conclusión. Lo único que se necesita, como he dicho, es ordenarla.

Pero cabe pensar en otras dos situaciones más complejas.

Cabe pensar, en primer lugar, en que en el origen tenemos una cantidad de información que es insuficiente para llegar a 
una determinada solución del problema. Ello puede ocurrir por que las premisas no ofrecen apoyo bastante, o bien porque no ofrecen ningún apoyo (la información que contienen es irrelevante para la conclusión). Y, en segundo lugar, es posible que en el punto de partida tengamos un exceso de información. Ello puede deberse, a su vez, a dos causas: a que la información es simplemente redundante, esto es, con menos información podría llegarse también a la conclusión; o a que la información de que se dispone en excesiva, pero no ya por redundante, sino por contradictoria. Las situaciones en que existe una información redundante no plantean, como es obvio, mayores problemas (el problema suele ser el de determinar que efectivamente hay una redundancia); podemos sin más reconducirlas a la situación (aproblemática) en que existe una cantidad de información necesaria y suficiente. Quedan, por tanto, dos tipos de situaciones problemáticas: cuando existe una información insuficiente, o bien contradictoria. En estos supuestos, argumentar sólo puede significar añadir nuevas informaciones, o bien eliminar informaciones existentes, de manera que la solución a la que se pretende llegar pueda tener, al final del proceso de la argumentación, un mayor apoyo (y, si fuera posible, un apoyo concluyente) que al comienzo. En estos casos sí que se puede decir que al final del proceso hemos obtenido algo nuevo, es decir, algo que no existía en un comienzo.

Por otro lado, si volvemos de nuevo al ejemplo de Sócrates, podemos imaginar que las situaciones problemáticas (por falta de información o por información contradictoria) podrían referirse a diversas cuestiones. Así, podría ofrecer dudas: 1) la validez del enunciado genera «»todos los hombres son mortales»; 2) la determinación del hecho de que «Sócrates es un hombre»; 3) el sentido en que deban entenderse las expresiones del enunciado general (cada una de ellas por separado: por ejemplo, «todos», «hombres», etc., o bien el conjunto, la proposición en bloque); 4) el que usemos o no en el mismo sentido la palabra «hombre» en las dos premisas. Veamos un poco más de cerca cómo podemos representar, en sus dos variantes, la primera situación.

Supongamos, en primer lugar, que no podemos partir sin más del enunciado general «todos los hombres son mortales», porque nuestro interlocutor en el diálogo lo pone en cuestión. Podemos sin embargo avanzar una afirmación como «la experiencia muestra que todos los hombres han muerto antes de los 170 años», y es bastante probable que nuestro interlocutor no tenga más remedio que asentir a ella. Si él no tiene nada que aducir en contra del enunciado general (o en favor de su nega- 
ción), el proceso de argumentación podríamos quizás representarlo así:

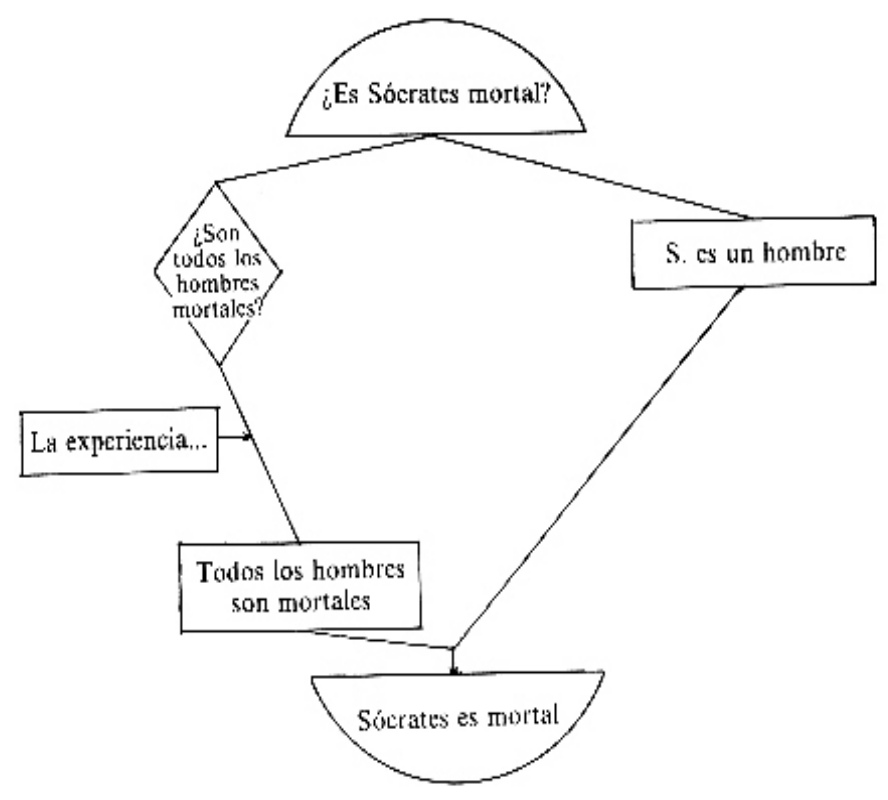

La segunda posibilidad era que la duda a propósito del enunciado general «todos los hombres son mortales» surja como consecuencia de una afirmación efectuada por un interlocutor y que la contradice; por ejemplo: «algunos filósofos son inmortales». En este caso, quien pretende argumentar en favor de la conclusión «Sócrates es mortal» podría aducir una afirmación como «no ha habido ningún filósofo que haya vivido más de 170 años». Suponiendo que nuestro interlocutor no ponga en cuestión esta afirmación (lo que seguramente no haría aunque él mismo fuera un filósofo) y no avance tampoco ninguna otra afirmación en favor de su primer aserto, podemos concluir negando esto último (la afirmación inicial de nuestro interlocutor). Ahora bien, al negarlo deja de existir la contradicción inicial, de manera que desaparece también la razón para dudar del enunciado general «todos los hombres son mortales». La argumentación podríamos representarla así: 


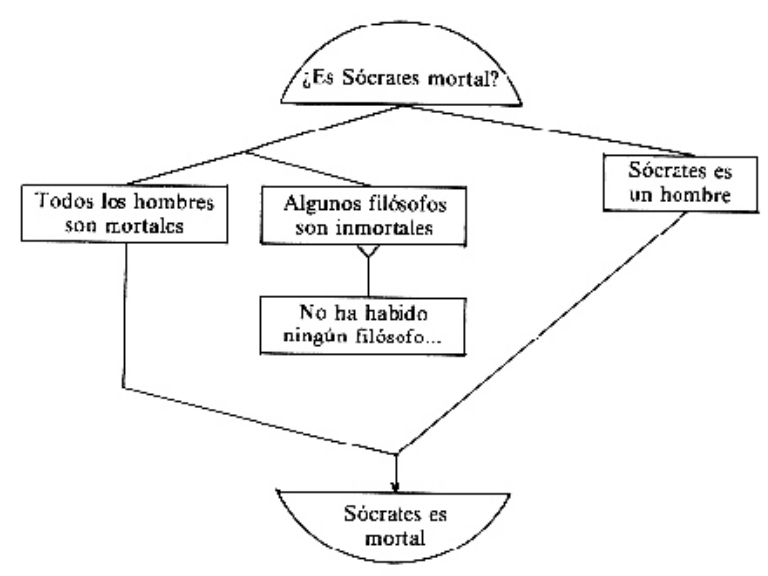

En el primer caso (información insuficiente) la afirmación de que «la experiencia muestra que todos los hombres han muerto antes de cumplir 170 años» nos permite pasar de la situación de duda inicial a la proposición «todos los hombres son mortales», de manera que lo primero es un argumento en favor de lo segundo. En el segundo caso (información contradictoria) la afirmación «no ha habido ningún filósofo que haya vivido más de 170 años» nos permite descartar una proposición (es un contraargumento respecto de esta última) y ratificar así la proposición contradictoria con ella. En los diagramas, el símbolo « $\longrightarrow$ 》 se utiliza, pues, en el sentido de «es un argumento en favor de», $\mathrm{y}$ «— argumento en contra de».

Ahora bien, las dos situaciones examinadas son todavía demasiado simples. Ni en la vida cotidiana ni, por supuesto, en la vida jurídica argumentar significa efectuar operaciones tan simples como las aquí representadas. ¿Para qué entonces sustituir la representación lógica habitual de los argumentos por diagramas de flechas como los aquí trazados?

\section{III}

La ventaja esencial de la representación propuesta [cfr. E. v. Savigny, 1976; U. Neumann, 1986; Schroth, 1980] es que de esta 
manera es posible dar cuenta de la relación «es un argumento en favor de» y «es un argumento en contra de» sin las que difícilmente se puede reconstruir el proceso real de las argumentaciones, esto es, las argumentaciones como realmente se producen. Esta relación difiere de la relación de inferencia lógica, al menos, en dos aspectos: 1) porque tiene un carácter más débil, en el sentido de que $x$ puede ser un argumento en favor de $y$ y $x$ ser verdadero (o, en general, válido) sin que por ello quede asegurada la verdad (la validez) de $y$; por ejemplo, porque $z$ es un argumento en contra de $y$ y que tiene mayor peso que $x ; 2$ ) porque tiene un carácter subjetivo, en cuanto que un argumento, según esto, es lo que una determinada persona considera como argumento.

La noción general de argumento ( « $x$ es un argumento») puede ahora, siguiendo a Eike von Savigny [1976, pág. 31; esta misma definición está también recogida por Schroth, 1980, pág. 120 y Neumann, 1986, pág. 115] introducirse así: «Un autor aduce $x$ como argumento en favor/en contra de algo, o aduce algo como argumento en favor/ en contra de $x$, y $x$ no se puede separar en partes, de manera que el autor aduzca la primera parte como argumento en favor/en contra de la segunda parte, o viceversa». Por otro lado, los argumentos pueden presentarse como argumentos independientes o en conexión con otros. Pueden así distinguirse las siguientes relaciones argumentativas [cfr. E. v. Savigny, ibid. pág. 39; Scroth introduce otros tipos de relaciones de las que aquí prescindo]:

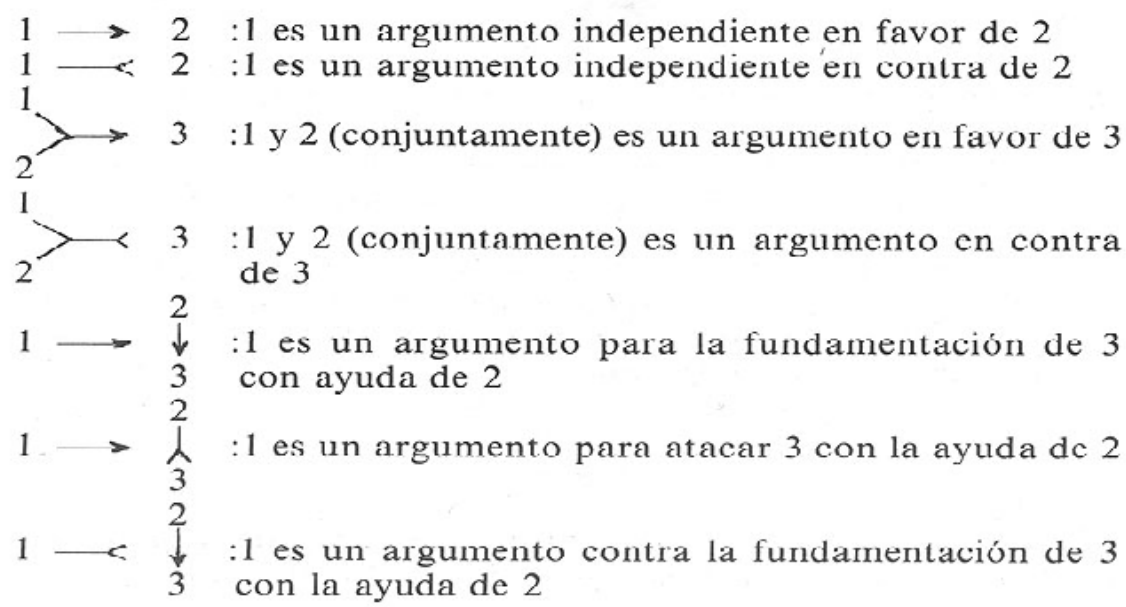


$1 \longrightarrow \hat{L}_{3}^{2}$ : 1 es un argumento contra el ataque de 3 con la

La tabla puede todavía, en mi opinión, enriquecerse en tres sentidos. Por un lado, introduciendo la noción de «negación de un argumento». Por ejemplo:

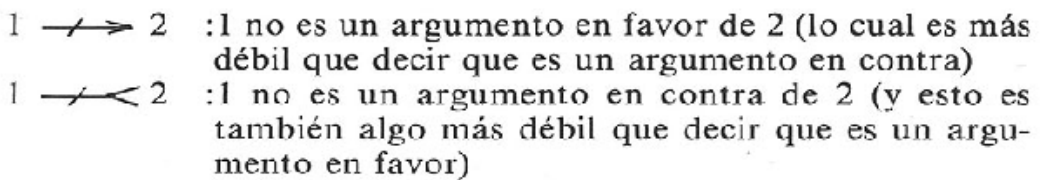
mento en favor)

Por otro lado, dos o más argumentos pueden constituir un argumento en favor o en contra de otro en forma conjuntiva o disyuntiva. Por ejemplo:

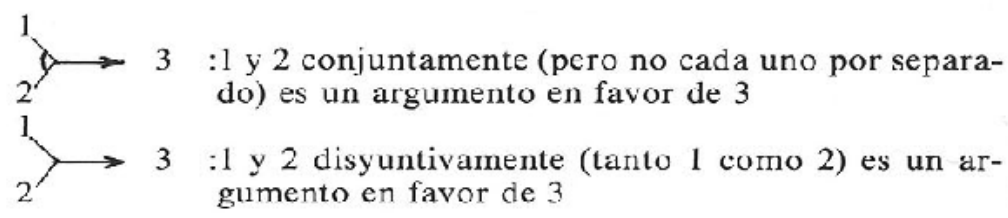

Finalmente, cabe introducir la noción de argumento deductivo que vendría a ser un caso específico (límite) de la de argumento en general. Como definición de argumento deductivo propongo la siguiente:

«Si un autor aduce $x$ como argumento en favor/ en contra de algo, o aduce algo como argumento en favor/en contra de $x$, entonces su argumento es un argumento deductivo si y sólo si:

a) $x$ no se puede separar en partes de manera que el autor aduzca la primera como argumento en favor/en contra de la segunda, o viceversa, y

b) si $x$ es un argumento en favor de y y $x$ (o, en su caso, lo aducido en favor de $x$ ) es válido (es verdadero, es correcto, etc.), entonces necesariamente debe serlo también $y$ (o, en su caso, $x$ ); y si $x$ es un argumento en contra de y y $x$ (o, en su caso, lo aducido en contra de $x$ ) es válido (es verdadero, es correcto, etc.), entonces necesariamente debe ser inválido (falso, incorrecto, etc.) $y$ (o, en su caso, $x$ ).» 
Los argumentos deductivos podríamos representarlos así:

$1 \longrightarrow 2: 1$ es un argumento deductivo en favor de 2

$1 \longrightarrow 2: 1$ es un argumento deductivo en contra de 2

En los diagramas utilizados en el apartado 2., el último tramo habría que considerarlo como deductivo. El segundo de los diagramas, por ejemplo, podría representarse ahora así:

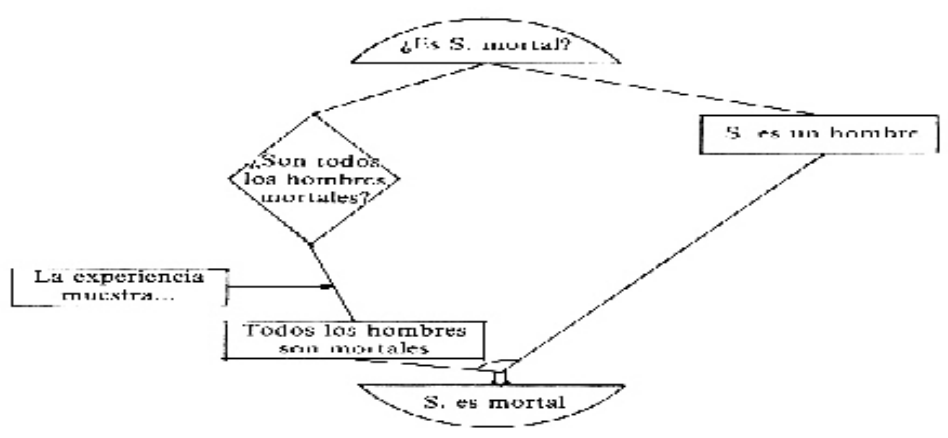

Antes de acometer la tarea de trasladar todo esto al campo de la argumentación jurídica, es preciso introducir todavía nuevas distinciones. En los ejemplos utilizados hasta ahora he recurrido a diversas figuras geométricas para distinguir tipos distintos de enunciados o, si se quiere, de actos de lenguaje que integran el acto de lenguaje complejo en que consiste argumentar. Hasta ahora hemos utilizado

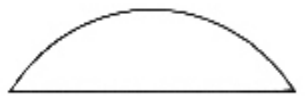

para representar el planteamiento de un problema 


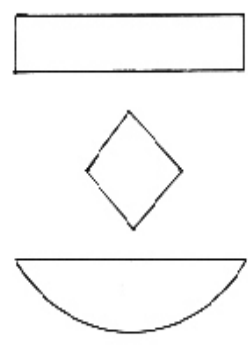

para representar enunciados empíricos (universales o singulares)

para representar una pregunta que se plantea en el curso de la argumentación y que puede tener una o más salidas

para representar la propuesta de una solución al problema inicial

Si queremos representar, sin embargo, adecuadamente argumentaciones no tan simples y, en particular, si queremos aplicar lo anterior a las argumentaciones jurídicas, debemos introducir nuevas categorías de enunciados. Básicamente [Rottleuthner,1980, por ejemplo, hace una clasificación en la que incluye diez categorías de enunciados] serían éstas:
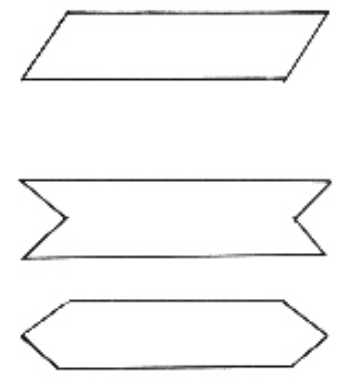

para representar un enunciado normativo (que obliga, prohíbe o permite hacer algo) que no sea el resultado final de la argumentación

para representar una definición o un postulado de significado

para representar un enunciado valorativo (de la forma «es deseable el estado de cosas $\mathrm{S} »)$

A partir de aquí podrían crearse todavía (si fuese necesario) subtipos de enunciados: por ejemplo, podría distinguirse entre enunciados empíricos universales o singulares, entre enunciados normativos abstractos y concretos, etc... Y podrían crearse también enunciados intermedios o híbridos. Por ejemplo:

para representar un enunciado deóntico ambiguo que pueda interpretarse tanto como una norma (lado derecho) como una proposición normativa (lado izquierdo) 

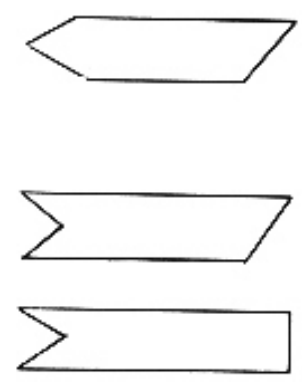

para representar un principio (que puede considerarse como un enunciado a mitad de camino entre los valores y las normas)

para representar una definición estipulativa

para representar una definición lexicográfica

$\mathrm{Y}$, en fin, podría distinguirse también entre todos los anteriores enunciados cuando se afirman categóricamente, o bien de forma simplemente hipotética. Así, por ejemplo:

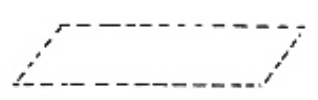

para representar un enunciado del tipo: «supongamos que existe una norma...»

para representar un enunciado del tipo: «supongamos que ha

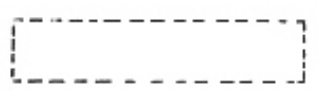
sido, es o será el caso de que...»

El conjunto de las anteriores relaciones de argumentación y de los tipos de enunciados que he introducido en este apartado permiten, me parece, representar adecuadamente cualquier argumentación jurídica que tiene lugar de hecho. En lo que sigue sólo haré uso, sin embargo, de un número relativamente reducido de estas categorías.

\section{IV}

Y ahora llega por fin el momento de ver cómo puede utilizarse lo anterior en el campo de la argumentación jurídica. El argumento a propósito de la inmortalidad de Sócrates era muy simple, pero puede ayudarnos, según creo, a introducirnos en el caso más complejo 
de los argumentos jurídicos.

Para ello, conviene empezar por establecer qué es lo que puede considerarse como un problema jurídico que, por tanto, puede desencadenar un proceso argumentativo. Si nos limitamos al campo de la aplicación del Derecho, podemos convenir quizás en que un problema jurídico comienza con una pregunta del tipo «¿cómo se debe solucionar el caso (real o ficticio) $\mathrm{x} »$ ?, para la que hay que encontrar una respuesta de la forma» al caso $\mathrm{x}$ debe aplicársele la solución y». Entre el problema y la solución tendremos que contar, por lo menos, con un enunciado empírico de la forma «x ha tenido lugar», y uno normativo que correlacione el caso y la solución: «en los casos X (y x está excluido en X) debe ser y»).

Antes de seguir adelante conviene todavía aclarar la noción de «caso» y de «solución» [sigo aquí a Alchourrón y Bulygin, 1973]. Los casos se definen a partir de propiedades y pueden ser abstractos o concretos. Por ejemplo, la muerte de A ocasionada por B, C y D en las circunstancias E, o la acción del señor $\mathrm{S}$ al decir en determinadas circunstancias «todos los soldados son asesinos potenciales» son casos concretos. El homicidio en riña tumultuaria y las injurias al ejército son casos abstractos. Los supuestos de hecho de las normas establecen casos abstractos y los problemas jurídicos pueden referirse a casos abstractos o concretos [en general puede decirse que la dogmática se centra en los primeros y los jueces y órganos aplicadores del Derecho en los segundos]. De forma parecida, las soluciones -que son enunciados que constan de una acción y de un operador deóntico- pueden ser también concretas o abstractas. La imposición a B, C y D de la pena de diez años y un día de prisión mayor o la absolución del Sr. S son soluciones concretas. La fijación de la pena de prisión mayor para el homicidio en riña tumultuaria o de prisión menor para las injurias al ejército son soluciones abstractas. Se puede entonces utilizar las minúsculas para referirse a casos o soluciones concretas (el caso x o la solución y) y las mayúsculas para aludir a casos abstractos y soluciones abstractas (el caso X o la solución $\mathrm{Y}$ ); se parte también de que $\mathrm{x}$ está incluido en $\mathrm{X}$ e y en Y. Los casos concretos, sin embargo, pueden considerarse como más complejos que los abstractos en cuanto que plantean por lo menos dos problemas que no se dan en los otros: el problema de determinar si efectivamente ha ocurrido $\mathrm{x}$; y el de fijar una solución concreta para el mismo: y. Si se parte, como aquí se hace, de casos concretos, el tipo de argumentación más simple podría representarse así: 


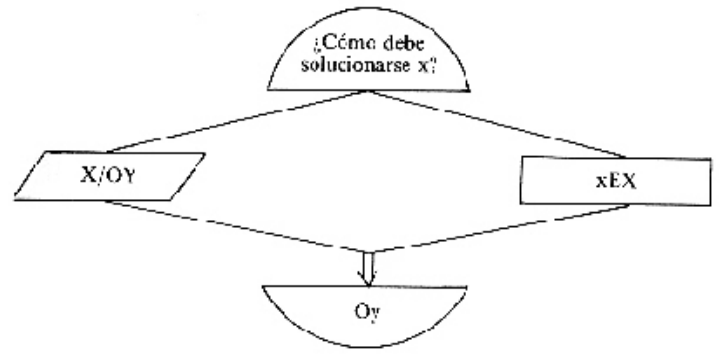

En el diagrama, O representa un operador deóntico, cualquiera que este sea. Por otro lado, se prescinde también -como igualmente haré en lo sucesivo- de la cuestión del paso de $\mathrm{Y}$ a $\mathrm{y}$. En realidad, esto último no significa simplificar demasiado las cosas, pues con frecuencia las soluciones previstas en las normas son ya soluciones inequívocamente determinadas, de manera que no hay mayor problema en pasar de la solución abstracta a la concreta; por ejemplo, cuando una norma establece que son nulos los matrimonios contraídos por error en la persona o por alguna circunstancia que vicie el consentimiento, o que el cese efectivo de la convivencia conyugal durante el transcurso de al menos cinco anos a petición de cualquiera de los cónyuges es causa de divorcio.

Ahora bien, en forma semejante a lo que sucedía en el ejemplo tradicional de silogismo, podría ocurrir ahora que nos encontráramos con problemas de falta de información o de información contradictoria, a propósito de las siguientes circunstancias:

1) Existen dudas sobre qué norma haya que aplicar al caso x. Llamaré a estos problemas [aquí, como en los otros tres supuestos, sigo a MacCormick, 1978, págs. 65 y sigs.] problemas de relevancia.

2) Existen dudas sobre de qué manera deben entenderse los términos (todos ellos o algunos de ellos) de la norma X/OY. Llamaré a estos problemas, problemas de interpretación.

3) Existen dudas sobre si $x$ ha tenido lugar. Llamaré a estos problemas, problemas de prueba.

4) Existen dudas sobre si $x$ es un supuesto de $X$. Llamaré a estos problemas, problemas de calificación. 
Esquemáticamente, podría representarse así:

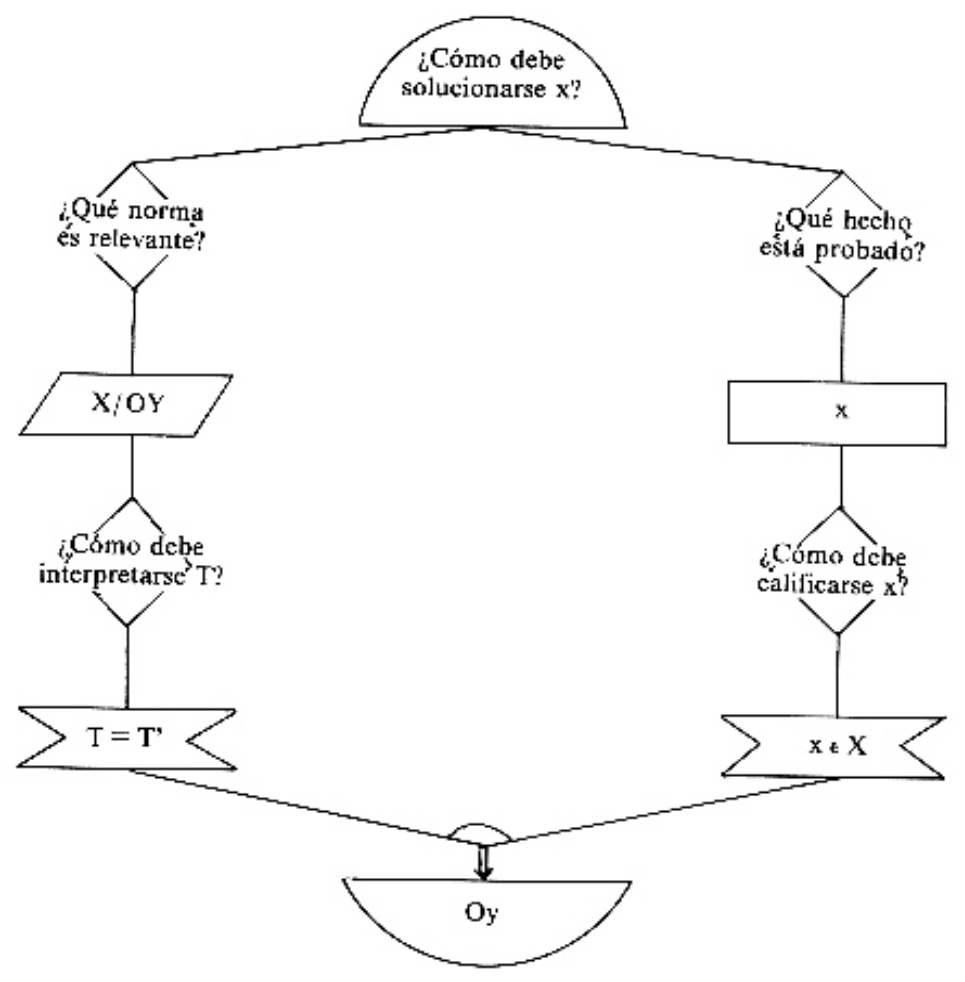

Evidentemente, la solución de algunos casos jurídicos puede requerir resolver problemas de los cuatro tipos o de más de un tipo de los señalados; a veces no es fácil determinar de qué tipo de problema se trata [en particular, puede discutirse si los problemas de calificación y de interpretación son en realidad problemas distintos; no entraré sin embargo aquí en esta cuestión], y casi siempre se producen remisiones de un tipo de problema a otro, de manera que la clasificación anterior vendría a establecer también algo así como tipos ideales de problemas jurídicos. Creo, sin embargo, que la misma constituye un buen esquema inicial para reconstruir conforme a él cualquier caso jurídico real. 
A partir de dicho esquema, puede pasarse a considerar la argumentación jurídica como un proceso (el proceso para solucionar un problema jurídico) en el que cabe distinguir los siguientes estadios:

1. Identificación del tipo de problema que hay que resolver. En lo que sigue voy a considerar únicamente los problemas de relevancia, si bien parto de la hipótesis de que los otros problemas son susceptibles de un tratamiento análogo.

2. Determinación de si se trata de una situación en que existe una insuficiencia de información (es decir, de si existe una laguna [2.1.]) o bien un exceso de información (una contradicción [2.2. $\left.{ }^{1}\right]$ ). Los problemas de redundancia, o bien son casos aproblemáticos (es decir, existe realmente redundancia) o bien pueden considerarse como casos de información insuficiente (que se transforma en redundante cuando se añaden premisas que se entienden implícitas).

3. Establecimiento de hipótesis, esto es, de nuevas premisas. Aquí deben introducirse las siguientes consideraciones:

3.1. Si se trata de un problema de insuficiencia de información, entonces es preciso efectuar un paso desde la información disponible (la norma dada: X'/OY) a la información que podría permitir resolver el caso (una nueva norma). Aquí cabe todavía efectuar tres hipótesis distintas, en cuanto que esa nueva norma podría consistir en:

3.1.1. Una norma de la forma $\mathrm{X}^{\prime} \mathrm{v} \mathrm{X} / \mathrm{OY}$, es decir, una norma que consista en una ampliación del supuesto de hecho respecto de la norma inicial y que contiene ya el caso $\mathrm{x}$.

3.1.2. La negación de esa norma, a la que llamaré negación externa (débil), y cuya forma sería -(X’vX/OY). Dicha negación viene a expresar que no existe, no cabe construir, no tiene vigencia, etc., una norma de la forma X'vX/OY.

3.1.3. Una norma de la forma $-\mathrm{X} /-\mathrm{OY}$, a la que llamaré negación interna (fuerte) de la norma $\mathrm{Y} / \mathrm{OY}$.

3.2. Si se trata de un problema de información contradictoria (por ejemplo, se parte de que existe una norma N1: X/OY y una norma N2: X/-OY), entonces el paso debe darse en el sentido de suprimir bien N1 o bien N2 (pero no las dos). Suprimir N1 significa confirmar o justificar N2, y viceversa.

4. Justificación de las hipótesis, lo que implica justificar el paso desde las premisas iniciales a las nuevas premisas. Aquí, de nuevo, es preciso distinguir diversos supuestos y efectuar varias consideraciones al respecto:

\footnotetext{
${ }^{1}$ Véase el esquema al final del trabajo.
} 
4.1. En el supuesto de laguna, los argumentos a efectuar para la justificación del paso en cuestión podrían llamarse argumentos analógicos en sentido amplio. Pero hay que distinguir todavía tres tipos de argumentos analógicos: la analogía en sentido estricto, la no analogía y el argumento e contrario. Dentro del primer tipo, es decir, dentro de los argumentos analógicos en sentido estricto, cabe hacer todavía una subdistinción entre argumentos a pari y a fortiori.

4. 1. 1. Lo que tienen en común estos dos tipos de argumentos analógicos en sentido estricto (argumentos a pari y a fortiori) es que se usan para llegar a un mismo resultado, esto es, para justificar una norma de la forma X'vX/ OY. El procedimiento de justificación difiere, sin embargo, en el siguiente sentido:

4.1.1.1. En los argumentos a pari, en favor del paso de X'/OY a X'vX/OY se aducen tres enunciados de la siguiente forma:

- Un enunciado que establece que los casos semejantes deben tener la misma solución jurídica. Llamaré a este enunciado principio de igualdad de trato (PI).

- Un enunciado empírico que establece que X y X' son semejantes en las propiedades A, B y C. Llamaré a este enunciado, enunciado de semejanza (ES).

- Un enunciado valorativo que establece que las propiedades en cuestión son las propiedades esenciales del caso. Llamaré a este enunciado, enunciado valorativo de relevancia (EVR).

4.1.1.2. En los argumentos a fortiori, cabe todavía establecer dos supuestos, según se trate de argumentos de menor a mayor o de mayor a menor:

4.1.1.2. 1. En los argumentos de menor a mayor, en favor del paso de X'/OY a $\mathrm{X}^{\prime} \mathrm{XX} / \mathrm{OY}$ se aducen tres enunciados de la siguiente forma:

- Un enunciado que establece que si está prohibido lo menos, está prohibido lo más. Por ejemplo: si está prohibido ir a más de $100 \mathrm{~km} / \mathrm{h}$ (=es obligatorio ir a menos de 100 $\mathrm{km} / \mathrm{h}$ ), cuando la carretera no esté iluminada (V), entonces está prohibido también ir a más de $100 \mathrm{~km} / \mathrm{h}$ cuando se circula por la noche y hay niebla (X). Llamaré a este enunciado, principio de prohibición ( $\mathrm{PPh})$.

- Un enunciado empírico que establece que $X$ tiene una propiedad que se daba en $\mathrm{X}^{\prime}$ (dificultades de visibilidad), en un grado mayor que Y. Llamaré a este enunciado, enunciado empírico de mayor intensidad (EEM). 
- Un enunciado valorativo que establece que una determinada propiedad (en el ejemplo, las dificultades de visibilidad) es una propiedad esencial del caso. Llamaré a este enunciado, enunciado valorativo de relevancia (EVR).

4.1.1.2.2. En los argumentos de mayor a menor, en favor del paso de X'/OY a $\mathrm{X}^{`} \mathrm{vX} / \mathrm{OY}$ se aducen tres enunciados de la siguiente forma:

- Un enunciado que establece que si está permitido lo más, entonces está permitido también lo menos. Por ejemplo, si en una carretera con dos carriles por cada lado (X) está permitido circular a más de $120 \mathrm{~km} / \mathrm{h}$, entonces si se trata de una autopista $(\mathrm{X})$ también está permitido ir a más de $120 \mathrm{~km} / \mathrm{h}$. Llamaré a este principio, principio de permisión (PP).

- Un enunciado empírico que establece que $X$ tiene una propiedad que se daba en X' (el peligro que supone circular por una carretera) en un grado menor que Y. Llamaré a este enun ciado, enunciado empírico de menor intensidad (EEN).

- Un enunciado valorativo que establece que la propiedad en cuestión es una propiedad esencial para el caso. Llamaré a este enunciado, enunciado valorativo de relevancia (EVR).

4.1.2. En el caso de la no analogía, hay que justificar que el paso que se pretende dar en 4.1.1. no es posible. Para ello bastará con rechazar alguno de los tres enunciados que constituyen (conjuntamente) la justificación en los supuestos de argumento a pari (4.1.1.1.) y de argumento a fortiori, bien se trate de argumentos de mayor a menor (4.1.1.2.1.) o de menor a mayor (4.1.1.2.2.). En la práctica, sin embargo, no parece que quepa rechazar los enunciados que enuncian principios (PI, PPh o PP), por lo que la argumentación se centrará en rechazar alguno de los enunciados empíricos (ES, EEM o EEN, en cada caso), o de los enunciados valorativos que establecen cuál es la propiedad relevante o esencial (EVR).

4.1.3. En el caso del argumento e contrario, en favor del paso de X'/ OY a -X'/OY hay que aducir dos enunciados de la siguiente forma:

- Un enunciado que afirma que lo no establecido expresamente en el supuesto de hecho de una norma jurídica debe resolverse en sentido contrario a dicha norma (o sea, negando la solución de la norma). Llamaré a este enunciado: principio de la interpretación restrictiva (IR). Este principio, a su vez, puede ser 
justificado apelando a otros principios o valores, como por ejemplo el de seguridad jurídica.

- Un enunciado empírico que establece que x no es un caso que caiga dentro de Y. Llamaré a este enunciado, enunciado empírico excluyente (EEE).

4.2. En los supuestos de información contradictoria, los argumentos a aducir para justificar alguna de las hipótesis (que aquí se reducen a dos aunque, naturalmente, podrían ser más de dos) consta de los siguientes enunciados que deben afirmarse conjuntamente:

- Un enunciado empírico que establece que NI (o bien N2) tiene como consecuencia ${ }^{2}$ un determinado estado de cosas. Llamaré a este enunciado: enunciado empírico consecuencialista (EEC).

- Un enunciado que establece que el estado de $\operatorname{cosas}^{3}$ en cuestión es indeseable. Llamaré a este enunciado, enunciado valorativo negativo (EVN).

- Un enunciado que establece la obligación de evitar situaciones que den lugar a juicios de valor negativos. Llamaré a este enunciado, principio de evitación de consecuencias negativas (PE).

5. El quinto y último estadio de la argumentación consiste en pasar de las hipótesis (de las nuevas premisas), una vez confirmadas o justificadas, a la solución. Las soluciones, en los diversos supuestos, serán:

5.1. En los supuestos de laguna.

5. 1. 1. En los casos de analogía en sentido estricto (argumentos a paro y a fortiori) $\mathrm{Oy}$, que es la consecuencia de aplicar la norma relevante $\mathrm{X}^{\prime} \mathrm{vX} / \mathrm{OY}$ al caso en cuestión, $\mathrm{x}$.

5.1.2. En los supuestos de analogía negativa no se llega a ninguna solución del problema. Habrá de nuevo que volver a plantear la cuestión e intentar ver si cabe la analogía en alguna otra dirección, o bien el argumento e contrario.

5.1.3. En el supuesto de argumento e contrario la solución será -Oy, que es la consecuencia de aplicar la norma relevante

$-X^{\prime} /$-OY al caso $\mathrm{x}$.

${ }^{2}$ El término «consecuencia» lo empleo en el más amplio sentido posible, incluyendo, por tanto, las consecuencias normativas.

${ }^{3}$ «Estado de cosas»incluye también, pues, los estados de cosas normativos. 
5.2. En los supuestos de contradicción (y suponiendo que la norma negada sea N1, lo que quiere decir que ha quedado confirmada N2), la solución será -Oy, que es la consecuencia de aplicar la norma relevante, $\mathrm{N} 2$ : $\mathrm{X} /-\mathrm{OY}$ al caso $\mathrm{X}$.

\section{V}

Todo lo visto en el anterior apartado puede sintetizarse en el siguiente esquema que, en mi opinión, ofrece una reconstrucción satisfactoria de la argumentación jurídica que se produce cuando hay que resolver problemas de relevancia. Dicho esquema -quizás con algunas modificaciones- podría extenderse también a los procesos de argumentación que surgen como consecuencia de problemas de interpretación, de prueba o de calificación.

Por lo demás, la principal conclusión de este trabajo es que en el Derecho (y quizás en cualquier otro campo) existen básicamente dos formas de argumentación, la analogía y la reducción al absurdo, y un número indeterminado de argumentos sustantivos, es decir, de enunciados o conjuntos de enunciados que cabe utilizar para la justificación de los pasos a dar en el contexto de estas dos formas y de las posibles combinaciones de estas dos formas. 


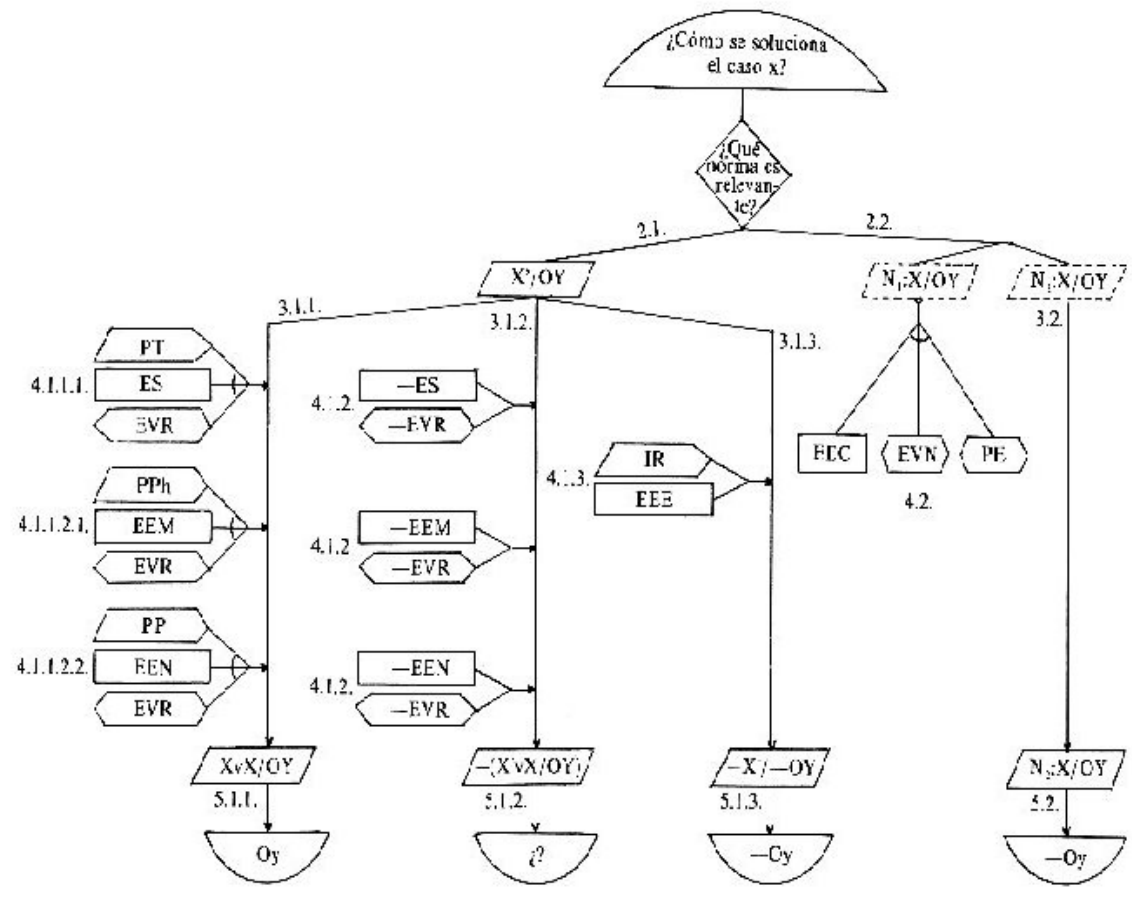




\section{BIBLIOGRAFÍA}

Aarnio, Aulis (1987), The rational as reasonable. A treatise on legal justification, Reidel, Dordrecht/Boston/Tokyo.

Alchourrón, Carlos, y Bulygin, Eugenio (1974), Introducción a la metodología de las ciencias jurídicas y sociales, Astrea, Buenos Aires.

Alexy, Robert (1989), Teoría de la argumentación jurídica, Madrid, Centro de Estudios Constitucionales (trad. de M. Atienza e I. Espejo de la ed. alemana de 1978: Theorie der juristischen Argumentation. Die Theorie des rationalen Diskurses als Theorie der juristischen Begründung, Suhrkamp, Frankfurt a.M.).

Lorenzen, Paul (1973), Pensamiento metódico, Sur, Buenos Aires.

MacCormick, Neil (1978), Legal Reasoning and legal Theory, Clarendom Press, Oxford.

Neumann, Ulfrid (1986), Juristische Argumentationslehre, Wissenschaftliche Buchgessellschaft, Darmstadt.

Patzig, Günther (1959), Die aristotelische Syllogistik, Vandenhoeck-Ruprecht, Göttingen.

Peczenik, Aleksander (1989), On law and reason, Kluwer Academic Publishers, Dordrecht/ Boston/ London.

Pereda, Carlos (1986), ¿Qué es una falacia?, en el libro colectivo Argumentación y filosofía, Cuadernos universitarios, Universidad Autónoma Metropolitana Iztapalapa, México.

Rottleuthrier, Hubert (1980), Plädoyer für eine empirische Argumentationstheorie, en «ARSN», cuaderno, nueva serie núm. 14, págs. 87-118.

Savigny, Elke von (1976), Argumentation in der Literaturwissenschaft.

Schroth, Ulrich(1980), Eine Methode derformalen Rekonstruktion von Gerichtsurteilen, en «ARSP», cuaderno, nueva serie núm. 14, págs. 119-126.

Toulmin, Stephen E. (1958), The uses of argument, Cambridge University Press.

Toulmin, Stephen E.; Rieke, Richard; Janik, Allan(1984), An introduc;ion to reasoning, MacMillan, New York, 2. ${ }^{\mathrm{a}}$ ed. (1. ${ }^{\mathrm{a}}$ ed., 1978). 